\title{
Effects of Lithium Doping and Post-processing on the Cathodoluminescence of Zinc Oxide Nanoparticles
}

\author{
K. E. McBean,* M. R. Phillips, ${ }^{*}$ and D. Drouin** \\ * Microstructural Analysis Unit, University of Technology, Sydney, PO Box 123, Broadway NSW \\ 2007 Australia \\ ** Université de Sherbrooke, 2500 Bd de l'université, J1K 2R1, Sherbrooke, Québec, Canada
}

Zinc oxide $(\mathrm{ZnO})$ is a wide band-gap $(3.37 \mathrm{eV})$ semiconductor with a large exciton binding energy of $60 \mathrm{meV}$. Because of these properties, $\mathrm{ZnO}$ is a good candidate for a wide range of applications, including varistors, phosphors as well as a large range of optical devices. Although the optical properties of $\mathrm{ZnO}$ have been extensively studied, there is still intensive debate as to the origin of a number of the luminescence centers observed.

In this study, $\mathrm{ZnO}$ nanoparticles were synthesized by mixing equal volumes of $0.05 \mathrm{M}$ zinc chloride $\left(\mathrm{ZnCl}_{2}\right)$ in ethanol and $0.20 \mathrm{M}$ sodium hydroxide (either $97+\%$ or semiconductor grade) in ethanol, for six hours at room temperature. To make lithium ( $\mathrm{Li}$ ) doped $\mathrm{ZnO}, 10 \mathrm{~mol} \%$ of $\mathrm{LiCl}$ was added to the $\mathrm{ZnCl}_{2}$ solution and the molar concentration of $\mathrm{ZnCl}_{2}$ was reduced accordingly. The resulting cloudy supernatant was then decanted, centrifuged, washed with double-distilled water followed by ethanol and then dried under vacuum. The resulting $\mathrm{ZnO}$ powders were identified as $\mathrm{ZnO}$ (synthesized with $97+\% \mathrm{NaOH}$ ), $\mathrm{HP} \mathrm{ZnO}$ (synthesized with semiconductor grade $\mathrm{NaOH}$ ) and $\mathrm{ZnO}: \mathrm{Li}$ (Li doped $\mathrm{ZnO}$ ). The affect of hydrogen on the cathodoluminescence (CL) properties of both $\mathrm{ZnO}$ and $\mathrm{HP} \mathrm{ZnO}$ was observed by annealing a portion of both these samples at $700^{\circ} \mathrm{C}$ for 10 minutes in an atmosphere of $5 \% \mathrm{H}_{2} / 95 \% \mathrm{Ar}$ and subsequently performing CL measurements of annealed and un-annealed samples. To observe the affects of Li doping on the $\mathrm{CL}$ of $\mathrm{ZnO}$, both unannealed and a portion of $\mathrm{ZnO}: \mathrm{Li}$ annealed at $700^{\circ} \mathrm{C}$ for 10 minutes in an inert atmosphere of argon, were observed.

Cathodoluminescence spectra and images were collected in a LEO Supra 55VP SEM using a Gatan MonoCL2 system equipped with a Hamamatsu R2228 PMT. A liquid helium circulation cold finger was used to cool the samples to approximately $6 \mathrm{~K}$ (see figures for exact temperatures). As seen in Fig. 1, un-annealed $\mathrm{ZnO}$ and $\mathrm{HP} \mathrm{ZnO}$ display a near band-edge (NBE) emission at $3.27 \mathrm{eV}$, which is greatly intensified after annealing in $\mathrm{H}_{2}$ (Fig. 2). Fig. 2 also shows the emergence of an additional peak, to the right of the NBE which is attributed to the affects of hydrogen. It can be seen from Fig. 3 , that this luminescence centre, at $373 \mathrm{~nm}$, has a different spatial distribution to the NBE emission $(377 \mathrm{~nm})$ and the longitudinal optical (LO) phonon replicas (385 nm, $394 \mathrm{~nm})$, which demonstrates that the emission at $373 \mathrm{~nm}$ can be attributed to a different recombination centre

The affect of Li doping on the luminescence of $\mathrm{ZnO}$ is of interest due to its use as a charge compensating ion when doping $\mathrm{ZnO}$ with tri-valent metal ions. Fig. 4 shows that Li doping results in a visible emission centered at $2.3 \mathrm{eV}$ in the un-annealed sample, but that annealing in Ar greatly enhances the NBE emission, and the visible emission is now observed at $2.5 \mathrm{eV}$. Ultimately, controlling the size of these particles (Fig. 5) will enable greater control of their luminescence and optimize their properties for a particular application. 


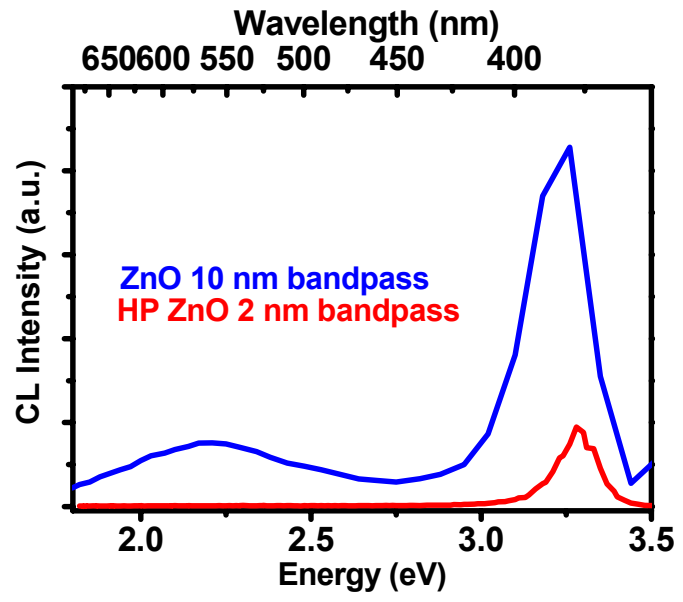

Fig. 1. CL spectra of un-annealed $\mathrm{ZnO}$ and $\mathrm{HP} \mathrm{ZnO}(5 \mathrm{kV}, 4.40 \mathrm{nA}, 5 \mathrm{~K})$

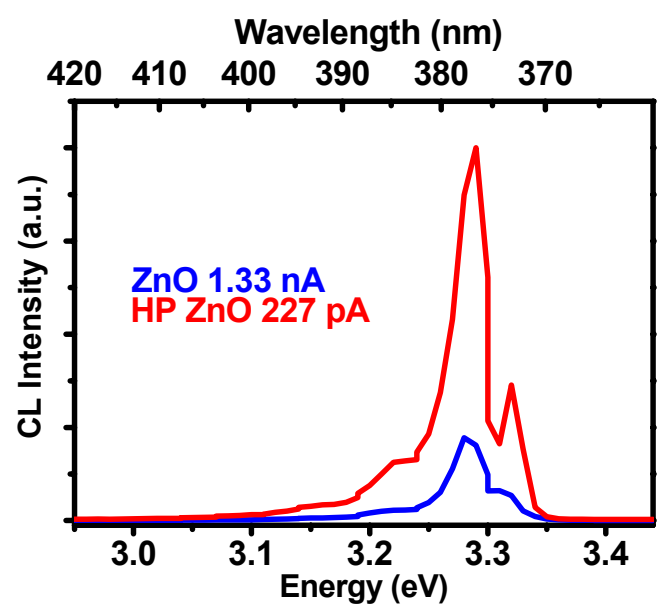

Fig. 2. CL spectra of $\mathrm{H}_{2}$ annealed $\mathrm{ZnO}$ and $\mathrm{HP}$ $\mathrm{ZnO}(5 \mathrm{kV}, 1 \mathrm{~nm}$ bandpass, $6 \mathrm{~K})$
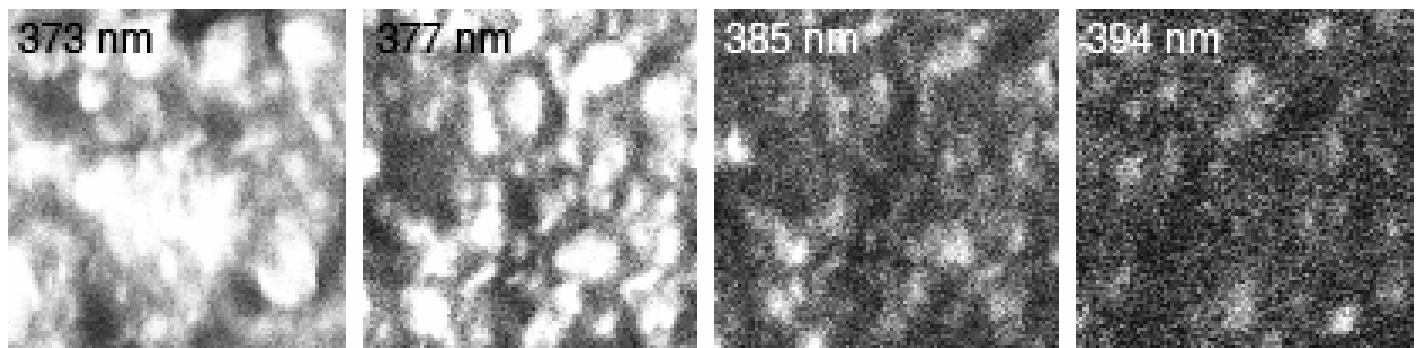

Fig. 3. Monochromatic CL images of $\mathrm{HP} \mathrm{ZnO}, \mathrm{H}_{2}$ annealed (WOF $=2.3 \mu \mathrm{m}, 5 \mathrm{kV}, 227 \mathrm{pA}, 1 \mathrm{~nm}$ bandpass, $5 \mathrm{~K}$ )

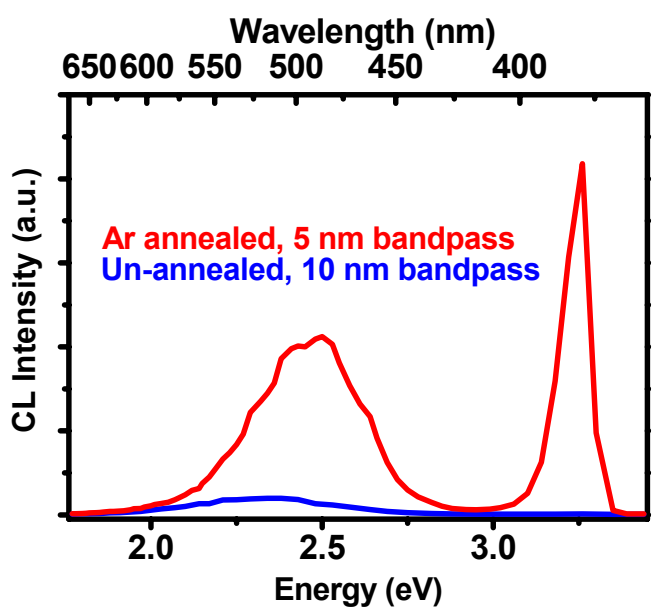

Fig. 4. CL spectra of un-annealed and Ar annealed $\mathrm{ZnO}: \mathrm{Li}(5 \mathrm{kV}, 4.40 \mathrm{nA}, 4.6 \mathrm{~K})$

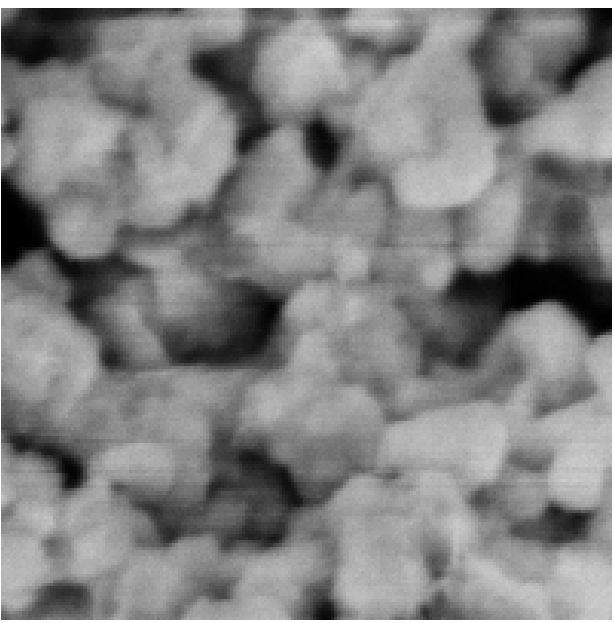

Fig. 5. Secondary electron image of un-annealed $\mathrm{ZnO}$ taken with in-lens detector $(\mathrm{WOF}=2.2 \mu \mathrm{m}$, $5 \mathrm{kV}$ ) 